Revue

de Sémantique

et Pragmatique
Revue de Sémantique et Pragmatique

38 | 2015

Le futur

\title{
Du rapport entre formes synthétique et analytique du futur - Etude de la variable modale dans un corpus oral micro-diachronique
}

\section{Lotfi Abouda et Marie Skrovec}

\author{
(2) OpenEdition \\ Journals \\ Édition électronique \\ URL : http://journals.openedition.org/rsp/512 \\ DOI : $10.4000 /$ rsp. 512 \\ ISSN : 2610-4377 \\ Éditeur \\ Presses universitaires d'Orléans \\ Édition imprimée \\ Date de publication : 1 septembre 2016 \\ Pagination : 35-57 \\ ISSN : 1285-4093 \\ Référence électronique \\ Lotfi Abouda et Marie Skrovec, « Du rapport entre formes synthétique et analytique du futur - Etude \\ de la variable modale dans un corpus oral micro-diachronique », Revue de Sémantique et Pragmatique \\ [En ligne], 38 | 2015, mis en ligne le 01 décembre 2016, consulté le 11 avril 2020. URL : http:// \\ journals.openedition.org/rsp/512 ; DOI : https://doi.org/10.4000/rsp.512
}

Ce document a été généré automatiquement le 11 avril 2020.

Revue de Sémantique et Pragmatique 


\title{
Du rapport entre formes synthétique et analytique du futur - Etude de la variable modale dans un corpus oral micro-diachronique
}

\author{
Lotfi Abouda et Marie Skrovec
}

\section{INTRODUCTION}

1 Le rapport en français moderne entre les formes analytique et synthétique du futur, appelées communément respectivement futur périphrastique (FP) et futur simple (FS), a fait l'objet de nombreuses études. On y voit s'affronter deux hypothèses principales : celle d'un changement en cours selon laquelle le FS céderait à terme la place à la forme périphrastique (Mauger (1968), Togeby (1982), Fleischman (1983), etc.) ; et l'hypothèse d'une complémentarité entre les deux tiroirs, chacun se spécialisant, et donc se maintenant dans des configurations linguistiques spécifiques. Sauf que la nature exacte de ces configurations varie considérablement selon les auteurs : d'aspecto-temporelle chez Jeanjean (1988) et Bilger (2001), la différence devient syntaxique chez Emirkanian \& Sankoff (1986), modale chez Confais (1990), Maingueneau (1994) et Laurendeau (2000), voire tributaire du registre de langue, comme chez Arrivé, Gadet \& Galmiche (1986), Rocchetti (2004), ou Collier (2013).

$2 \mathrm{Si}$, au cours des dernières décennies, quittant le champ de débats strictement théoriques, de nombreux travaux sur corpus, oraux ou écrits, quantitatifs ou non (Emirkanian \& Sankoff 1986, Jeanjean 1988, Bilger 2001, Fleury \& Branca 2010, etc.) ont vu le jour, les corpus sur lesquels ces études se sont fondées nous paraissent à la fois relativement restreints sur le plan quantitatif, et surtout, de nature synchronique, peu adaptés à l'étude de la relation diachronique FP/FS.

Or, depuis peu, les chercheurs disposent d'un corpus oral susceptible de pallier ce manque de données pertinentes : les Enquêtes sociolinguistiques à Orléans (ESLO) ${ }^{1}$. 
Outre sa taille importante (actuellement autour de 7 millions de mots) qui permet la composition d'un sous-corpus équilibré, ESLO a en effet la particularité d'avoir été collecté en deux phases, à 40 ans d'intervalle, donnant ainsi naissance à deux souscorpus comparables, mais diachroniquement différenciés, ESLO1 (1968-1971) et ESLO2 (2008-). Nous entendons contribuer à l'étude du rapport FP/FS en nous basant sur un sous-corpus oral micro-diachronique d'un million de mots, constitué à parts égales d'ESLO1 et d'ESLO2.

Les 3370 occurrences du futur (FS et FP) identifiées dans ce corpus représentent une masse considérable de données, dont l'examen exhaustif dépasse largement le cadre de ce travail, d'autant que les types de relations (complémentarité, concurrence, ou évolutions indépendantes des deux tiroirs) coexistent et semble reliés à des paramètres divers (types d'emploi, aspect lexical, contextes d'emplois, etc.).

Dans cette étude, nous nous proposons de nous focaliser sur l'étude de la relation micro-diachronique FS/FP en relation avec la variable modale. En effet, non seulement c'est dans le champ de la modalité que la plupart des travaux cherchent à trouver une explication à la relation de concurrence ou de complémentarité entre le FS et le FP (Confais 1990, Wilmet 2007, Fleischman 1982, etc.), mais aussi, ainsi que nous le verrons, les données statistiques dont nous disposons montrent clairement que, si l'évolution micro-diachronique du FP/FS nécessite l'examen précis d'un nombre important de variables, c'est au niveau de l'évolution modale que les données nous paraissent a priori les plus significatives.

6 Après une première partie méthodologique, où il sera notamment question de présenter les données et la démarche suivie pour leur exploitation ainsi que la présentation et la justification de l'annotation, nous aborderons dans la deuxième partie quelques-unes des tendances statistiques jugées les plus pertinentes dans la distribution FP/FS et son évolution micro-diachronique d'ESLO1 à ESLO2. En troisième partie, pour tenter d'identifier le type de rapport microdiachronique entre le FP et le FS, nous nous proposerons de l'examiner de plus près par le biais de la variable modale, en explorant une couche d'annotation supplémentaire explicitant les types de modalités à l'œuvre.

\section{MÉTHODOLOGIE : CONSTITUTION DU CORPUS ET DÉMARCHE D'ANNOTATION}

7 La démarche d'analyse, prise dans sa globalité, peut être considérée comme basée sur corpus (data based dans la conception de Biber 2009). Il s'agit en effet de rechercher, dans un ensemble délimité de données langagières, des formes à propos desquelles des hypothèses ont été formulées et que nous nous proposons d'examiner. Néanmoins, l'approche s'inspire également de la méthodologie dite guidée par les données (datadriven pour Biber 2009), puisque, une fois les formes identifiées, nous menons une analyse sémantique inductive qui fait émerger le sens à partir de la seule observation $\mathrm{du}$ corpus. Nous verrons ainsi que les types d'emplois identifiés lors de la phase d'annotation ne correspondent pas toujours aux types reconnus habituellement dans les travaux antérieurs.

8 Ces choix sont déterminés entre autres par l'existence même du corpus ESLO, qui permet de comparer des données de même type collectées à 40 ans d'intervalle, et d'y 
examiner l'évolution des usages du FS et du FP du point de vue de la nature et de la fréquence des occurrences, en évitant le papillonnage.

9 La méthode, naturellement, a ses écueils, ayant trait au problème de la nécessaire délimitation d'un corpus de travail, qui induit un certain "angle mort" dans la description des usages. Même si les occurrences décrites ici sont d'une grande diversité, il est évidemment tout à fait possible que certains types d'emplois attestés par ailleurs ne figurent pas dans cette étude. De même, il est à envisager que d'autres tendances, potentiellement contradictoires, puissent être dégagées dans un corpus différent, basé sur des données écrites par exemple, dans la mesure où les genres discursifs représentés dans notre corpus sont nécessairement limités. Cet angle mort nous semble cependant être compensé par plusieurs avantages, comme celui de la visibilité accordée aux phénomènes fréquents et aux grandes tendances dans de vastes domaines du vernaculaire, ce qu'une approche exclusivement data-based ne saurait saisir, puisqu'elle consiste davantage à vérifier l'existence de phénomènes en glanant des occurrences à partir de différentes sources. En outre, la méthodologie choisie impose de considérer les données dans toute leur réalité et complexité : dans la tâche de catégorisation de toutes les occurrences par types d'emploi, qui n'en est que plus ardue, notre démarche oblige à examiner non seulement les types catégoriels, mais encore leurs frontières, et donc de considérer des emplois mixtes ou hybrides (cf. 1.3.).

\section{LE SOUS-CORPUS}

Le sous-corpus sur lequel s'appuie cette étude est un corpus échantillonné d'environ 1 million de mots ( 80 heures et 51 minutes), constitué, à parts égales, d'extraits d'ESLOl et d'ESLO2. Outre cet équilibrage diachronique, les deux extraits ESLO1/ESLO2 sélectionnés sont également comparables sur le plan qualitatif. S'agissant d'un corpus de données situées - les enregistrements transcrits sont assortis de métadonnées, qui renseignent sur le profil du locuteur -, nous avons veillé à un équilibrage diastratique, en termes de profil de locuteurs (une centaine environ), choisis selon les variables de sexe, d'âge et de catégorie socio-professionnelle ${ }^{2}$. Afin de maintenir la comparabilité ${ }^{3}$ entre les deux sous- corpus, il n'était pas en revanche possible, au moment où nous avons constitué ce corpus, d'assurer un équilibre diaphasique. Dans un corpus majoritairement constitué d'entretiens, nous avons toutefois réussi à intégrer, à hauteur de $20 \%$, deux genres interactionnels de "contrôle", i.e. les repas et les conférences.

\begin{tabular}{|l|l|l|l|l|}
\hline & Genre & ESLO1 & ESLO2 & Corpus global \\
\hline Durée (en min) & Conférences & 192 & 186 & 378 \\
\hline & Repas & 196 & 201 & 397 \\
\hline & Entretiens & 2042 & 2034 & 4076 \\
\hline & Total & 2430 & 2421 & 4851 (80h et 51min) \\
\hline Nombre de locuteurs & Entretiens & 30 & 30 & 60 \\
\hline
\end{tabular}




\section{DÉMARCHE}

12 Pour exploiter le corpus constitué, nous avons utilisé un logiciel d'analyse textométrique, $\mathrm{TXM}^{4}$, qui permet de lemmatiser un corpus transcrit et de l'étiqueter en parties du discours (pos) sous Treetagger ${ }^{5}$. Deux requêtes séparées ont été nécessaires pour extraire les occurrences du futur simple et celle du futur périphrastique ${ }^{6}$. Les listes d'occurrences obtenues ont par la suite été exportées dans des tableaux CSV, afin d'y être corrigées manuellement (l'objectif était d'écarter les erreurs d'annotation, mais aussi, en ce qui concerne le FP, d'exclure les occurrences d'aller au sens lexical plein de verbe de mouvement). Après correction, nous avons procédé à une annotation affinée, où chacune des occurrences a été sous-spécifiée pour un certain nombre de traits.

13 Corrigées et annotées, les listes d'occurrences, ainsi que les étiquettes d'annotation que nous avons ajoutées, ont par la suite été réinjectées sous TXM afin d'être exploitées en textométrie et permettre de croiser les approches quantitative et qualitative.

14 En effet, si nous nous sommes appuyés, pour effectuer les annotations, sur les questionnements actuels autour du futur, les annotations ont constitué elles- mêmes un nouveau point de départ pour une exploration quantitative des données. Mais à leur tour, les tendances quantitatives dégagées, si elles sont quelquefois significatives en elles-mêmes, obligent souvent, pour être correctement interprétées, à un retour à des analyses qualitatives où il est question d'identifier les éventuelles variables distributionnelles et co-textuelles (négation, personne, adverbes, etc.) pertinentes dans la distribution $\mathrm{FP} / \mathrm{FS}^{7}$.

\section{ANNOTATION}

15 La tâche d'annotation des types d'emplois et sous-spécifications a été réalisée entièrement manuellement et les étiquettes ont été attribuées à chacune des 3370 occurrences de FS ou FP après consultation non seulement du contexte de transcription élargi, mais aussi, très fréquemment, de l'extrait audio correspondant.

Pour les principales couches d'annotation, nous avons procédé par étapes. Une première phase de travail a été menée conjointement par nous deux dans le but de forger des étiquettes communes "de travail". Il n'était pas en effet pour nous question de partir d'une taxinomie déjà établie, mais de partir, dans cette phase descriptive, de l'effet de sens précis qui se dégage de l'occurrence. L'examen commun de plusieurs centaines de cas nous a permis d'harmoniser nos vues, non seulement en ce qui concerne les étiquettes proposées, interrogées constamment au cours de la phase d'annotation, mais aussi concernant les stratégies d'identification de ce qui relève de l'apport sémantique du marqueur étudié, pour éviter le risque permanent d'une confusion entre la valeur sémantique d'une occurrence temporelle avec son contexte d'apparition. Au cours d'une deuxième phase de travail, chacun d'entre nous a entrepris d'annoter individuellement les occurrences restantes, avant une dernière phase où il était question de confronter les deux annotations. Si l'accord interannotateurs a été assez élevé, un certain nombre de cas ont nécessité de longues discussions avant de parvenir à une annotation unifiée. 

ESLO2_REP_01_02_C_ 16
tu as fini avec tes lunettes là ? tu vas les casser recherche : le type d'emploi et la sous-spécification. occurrences en question'.

ESLO 1_REPAS_273_B_10

(1) va dire ça à mon frère l'impératif qui pourrait en être la glose (dis-lui ce soir). modale :

Deux étiquettes d'annotation seront principalement mobilisées au cours de la présente

La première étiquette spécifie le type de valeur sémantique exprimée par l'occurrence $\mathrm{du}$ futur, et peut recevoir une des trois valeurs possibles correspondant à l'une des trois classes d'emplois distinguées : en plus des deux emplois habituellement reconnus ${ }^{8}$, i.e. les emplois modaux (étiquetés “m”), et les emplois temporels (étiquetés "f", sans distinction, à ce stade, entre l'expression de la prospection et celle de futurité), il nous a paru très vite incontournable de reconnaitre une troisième classe, mixte, d'emplois (étiquetés “fm"). La reconnaissance de cette troisième classe d'emplois est l'une des implications directes de notre méthodologie de travail, i.e. une approche guidée par les données. Celle-ci nous conduit à étiqueter toutes les occurrences, y compris celles qu'on aurait sans doute écartées dans le cadre d'une analyse simplement basée sur corpus, parce qu'elles n'auraient pas correspondu aux exemples typiques qu'on aurait cherchés pour illustrer des classes d'emplois préétablies. Or il nous a paru très vite impossible d'inclure ces emplois mixtes dans l'une des deux classes dichotomiques habituelles sans sacrifier l'une des deux faces sémantiques exprimées par les

Ainsi, dans l'exemple suivant, le futur simple est à la fois futural et déperformatif :

ben tu lui diras ce soir qu'il faut qu'il mette un coton avec de l'éther

Emploi “de frontière", il recevra l'étiquette "fm" qui nous semble bien correspondre à son statut hybride ${ }^{10}$. Cette analyse s'oppose ainsi à une vision, sans doute majoritaire même si elle n'est pas toujours explicitement revendiquée, dans laquelle les temps ne reçoivent une interprétation modale que lorsqu'ils ont cessé de fonctionner comme marqueurs chronologiques ${ }^{11}$. Or, dans un exemple comme celui ci-dessus, l'emploi comme déperformatif repose précisément sur l'utilisation d'un FS qui ne se contente pas de localiser le procès dans le futur mais permet à l'énonciateur de formuler l'équivalent d'une injonction sans recourir à une forme injonctive explicite, comme

21 Dans cet autre exemple, le futur périphrastique combine une dimension futurale et

Exprimant un avertissement, ce futur périphrastique situe un évènement indésirable dans un futur irrémédiable ${ }^{12}$ si l'action en cours, qui en est la cause, ne s'arrête pas immédiatement. L'effet de sens d'avertissement résulte d'une inférence pragmatique déclenchée par cet emploi spécifique du FP dans lequel s'imbriquent futurité ${ }^{13}$ (le FP localise le procès dans l'avenir) et modalité (l'actualisation du procès se fait dans un 
avenir à la fois possible et inéluctable dans le cadre d'une implication dont l'antécédent est implicite : si tu continues comme ça, tu vas les casser).

La sous-spécification, seconde étiquette mobilisée lors de la présente recherche, concernera ici tous les emplois modaux, soit 176 occurrences de FS et 455 occurrences de FP. Cette couche d'annotation vise à identifier le type de modalité exprimée par les cas étiquetés " $m$ ". L'ensemble de l'annotation jusqu'à cette étape peut être présenté

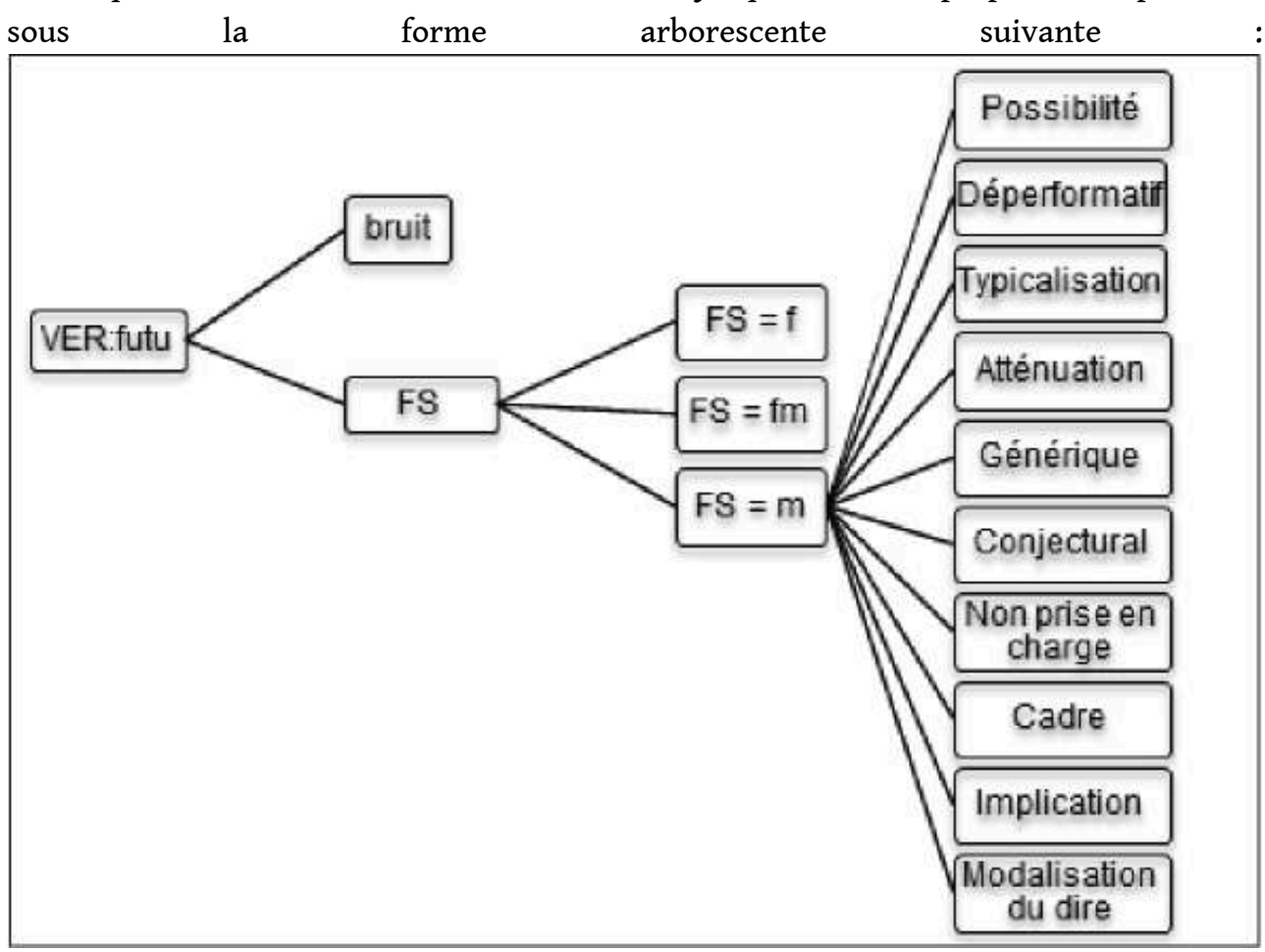

Figure 1 : Schéma arborescent du système d'annotation du futur simple

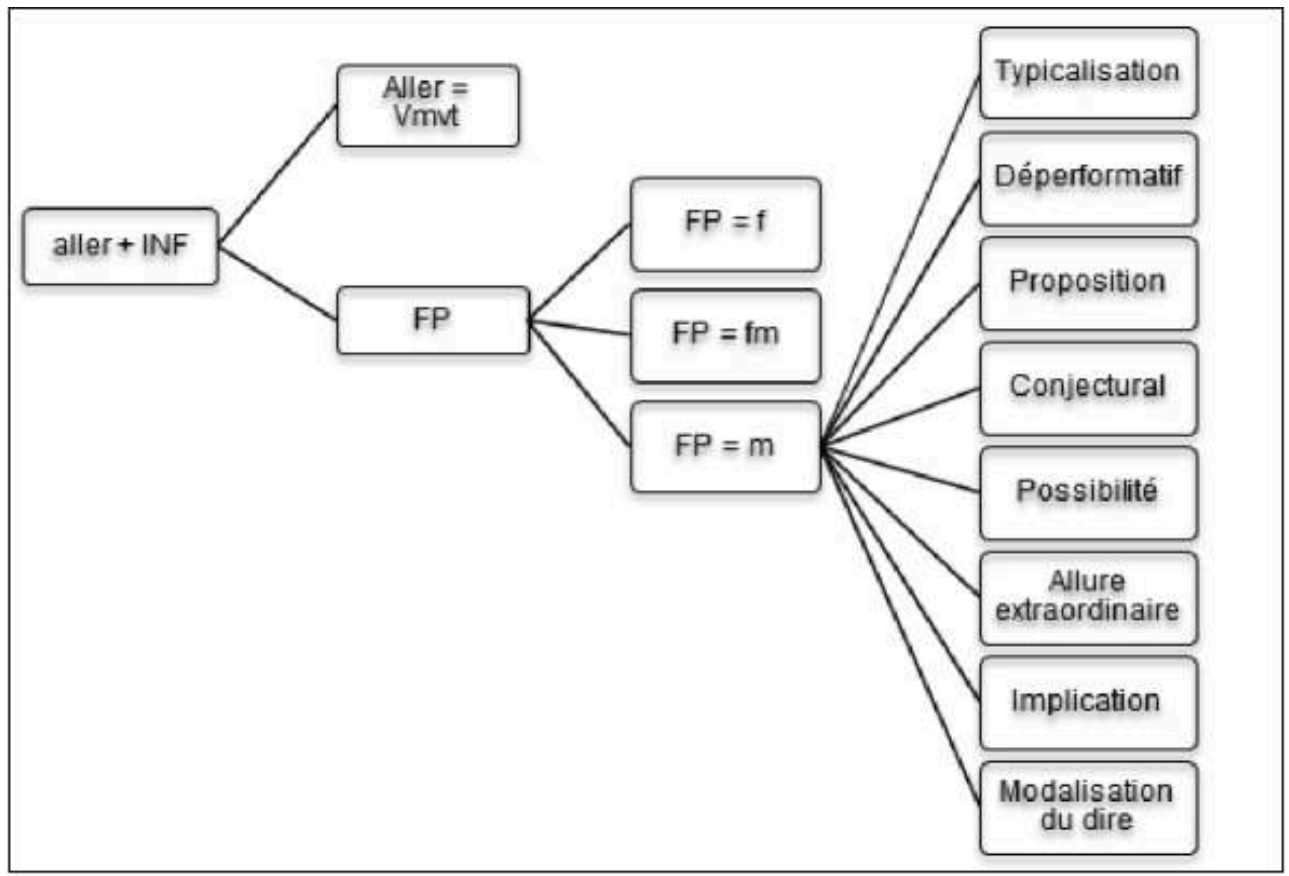

Figure 2 : Schéma arborescent du système d'annotation du futur périphrastique 


\section{ANALYSE QUANTITATIVE : QUELQUES TENDANCES EN MICRO-DIACHRONIE}

Une fois réintégrées dans txm, les différentes propriétés annotées (types d'emplois, sous-spécifications) ont constitué des variables pouvant faire l'objet de différentes requêtes en corrélation avec d'autres variables distributionnelles disponibles sur txm (négation, personne, modifieurs aspecto-temporels et modaux), ainsi qu'avec les métadonnées du corpus (ESLO1 vs ESLO2, genres interactionnels, tranche d'âge, CSP, etc.).

Nous nous proposons dans cette partie de nous focaliser sur quelques-unes des tendances quantitatives les plus significatives en micro-diachronie.

Présentées dans le tableau suivant:

\begin{tabular}{|l|l|l|l|}
\hline & ESLO1 & ESLO2 & ESLO1+2 \\
\hline FS & 985 & 466 & 1451 \\
\hline FP & 715 & 1204 & 1919 \\
\hline Total & 1700 & 1670 & 3370 \\
\hline
\end{tabular}

Tableau 2 : fréquence des occurrences du FS et du FP dans ESLO 1 et 2

les premières données quantitatives nous paraissent intéressantes. Elles montrent que si le nombre d'occurrences du futur (en tant que catégorie générique regroupant les deux tiroirs étudiés) reste remarquablement stable d'ESLOl à ESLO2, la répartition interne entre le FS et le FP s'inverse totalement ${ }^{14}$, comme on peut le visualiser sur le graphique suivant :

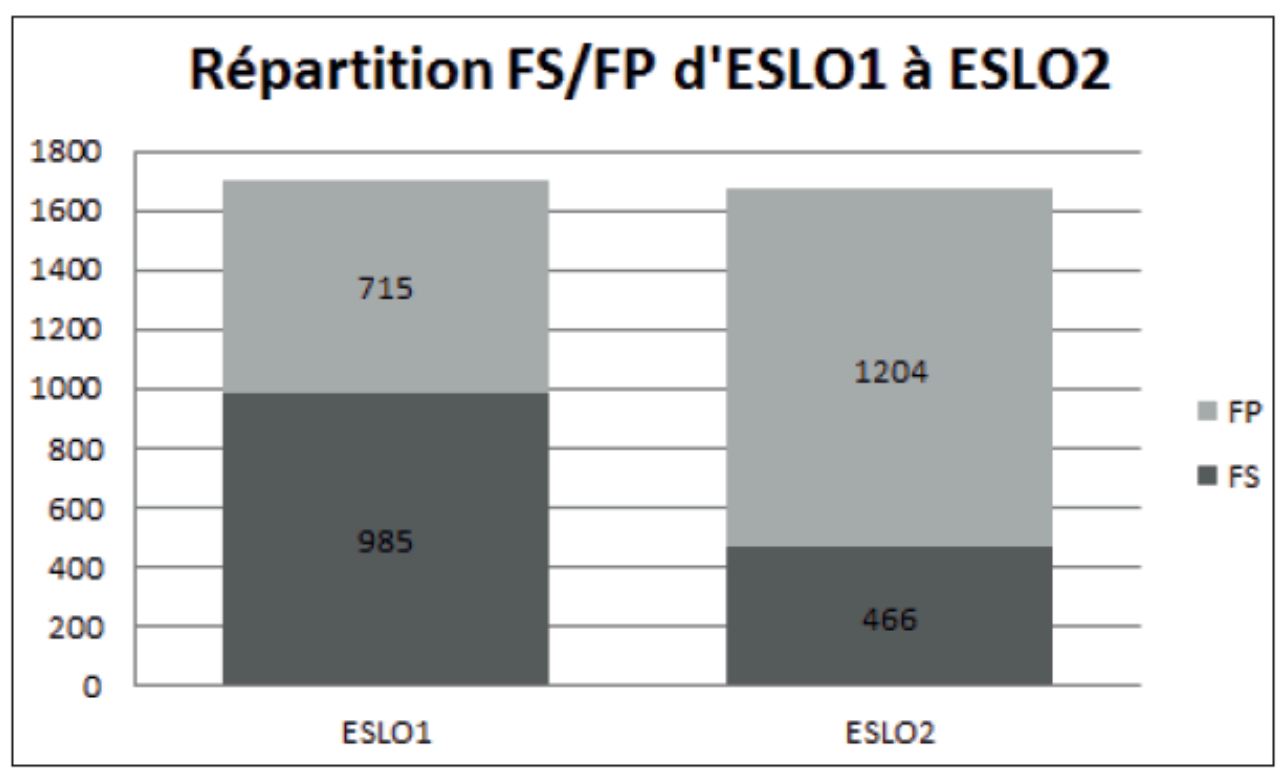

Vu la quasi stabilité, d'ESLOl à ESLO2, du nombre d'occurrences du futur (FS et FP compris), on pourrait être tenté de corréler les deux phénomènes statistiques observés, 
à savoir la forte chute du FS et la forte augmentation du FP. Toutefois, il nous parait indispensable d'examiner les tendances de plus près, en essayant, dans un premier temps, d'interpréter qualitativement la répartition entre les deux tiroirs en fonction des types d'emplois.

Comparons, dans un premier temps, pour chacune des deux formes les emplois purement chronologiques aux emplois contenant un trait modal (typés aussi bien $\mathrm{m}$ que $\mathrm{fm}$ ). En ce qui concerne le FP (cf. figure ci-dessous), nous constatons une progression importante aussi bien dans ses emplois temporels $(+67 \%)$ que dans ses emplois modaux $(+71 \%)$, et dans des proportions assez comparables. On peut a priori parler de progression homogène ${ }^{15}$.
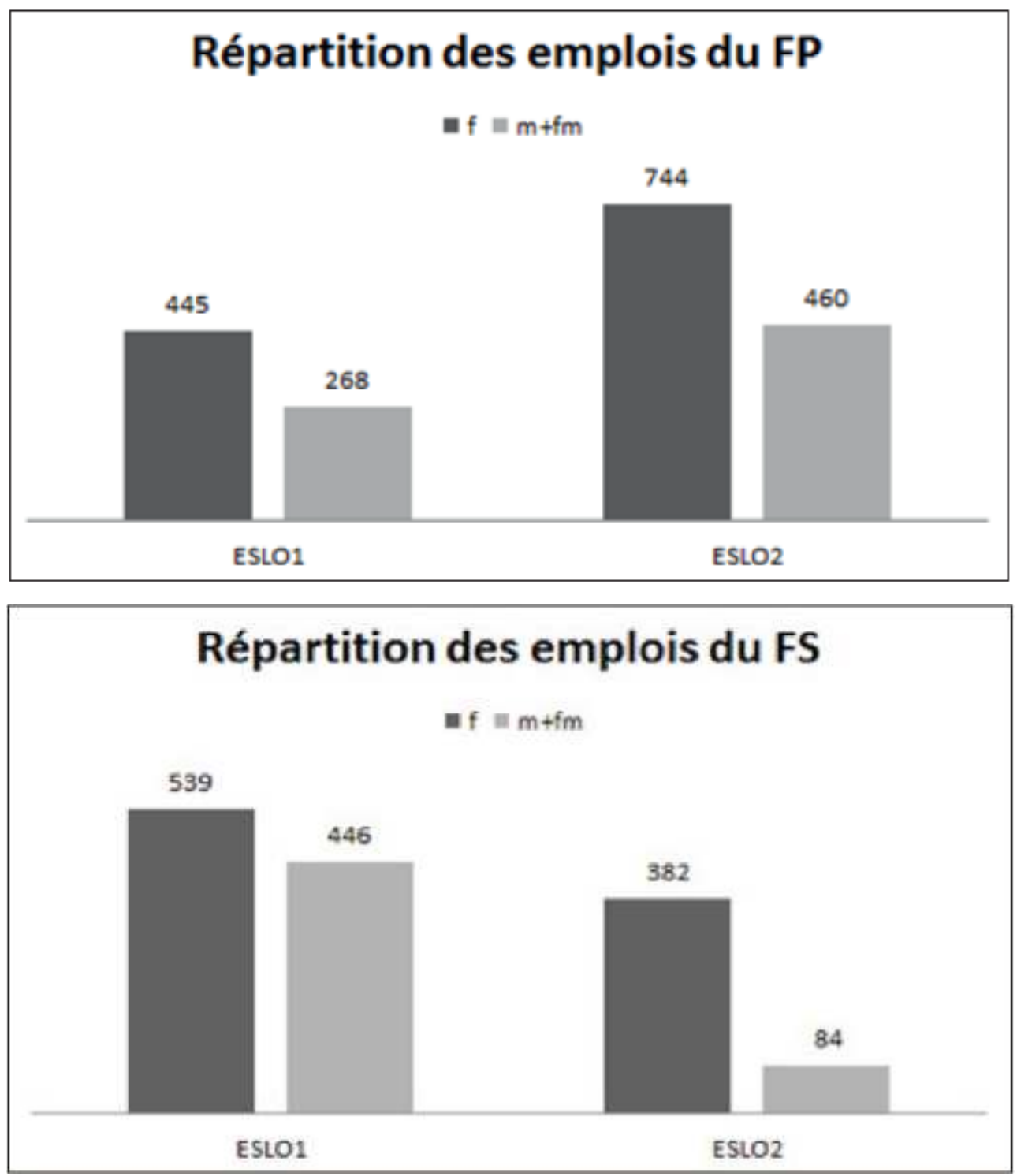

Figure 4 : Répartition des emplois du FP en nombre d'occurrences En ce qui concerne le FS, en revanche, on observe une chute pour les deux familles d'emplois, avec toutefois un recul particulièrement frappant dans ses emplois modaux, qui chutent de $81 \%$ en passant de 446 occurrences dans ESLO1 à 84 dans ESLO2 (tandis que les emplois temporels ne reculent que de $30 \%$ ) :

Cette chute importante des emplois modaux du FS, parallèlement à la forte augmentation du même type d'emplois pour le $\mathrm{FP}$, nous incite à examiner de plus près 
la répartition, en recourant à une troisième couche d'annotation, détaillant les sousspécifications attribuées aux formes étiquetées $\mathrm{m}$. Un décompte général de la répartition $\mathrm{FS} / \mathrm{FP}$ pour chaque sous-spécification de met en évidence les rapports suivants dans ESLO1 puis ESLO2:

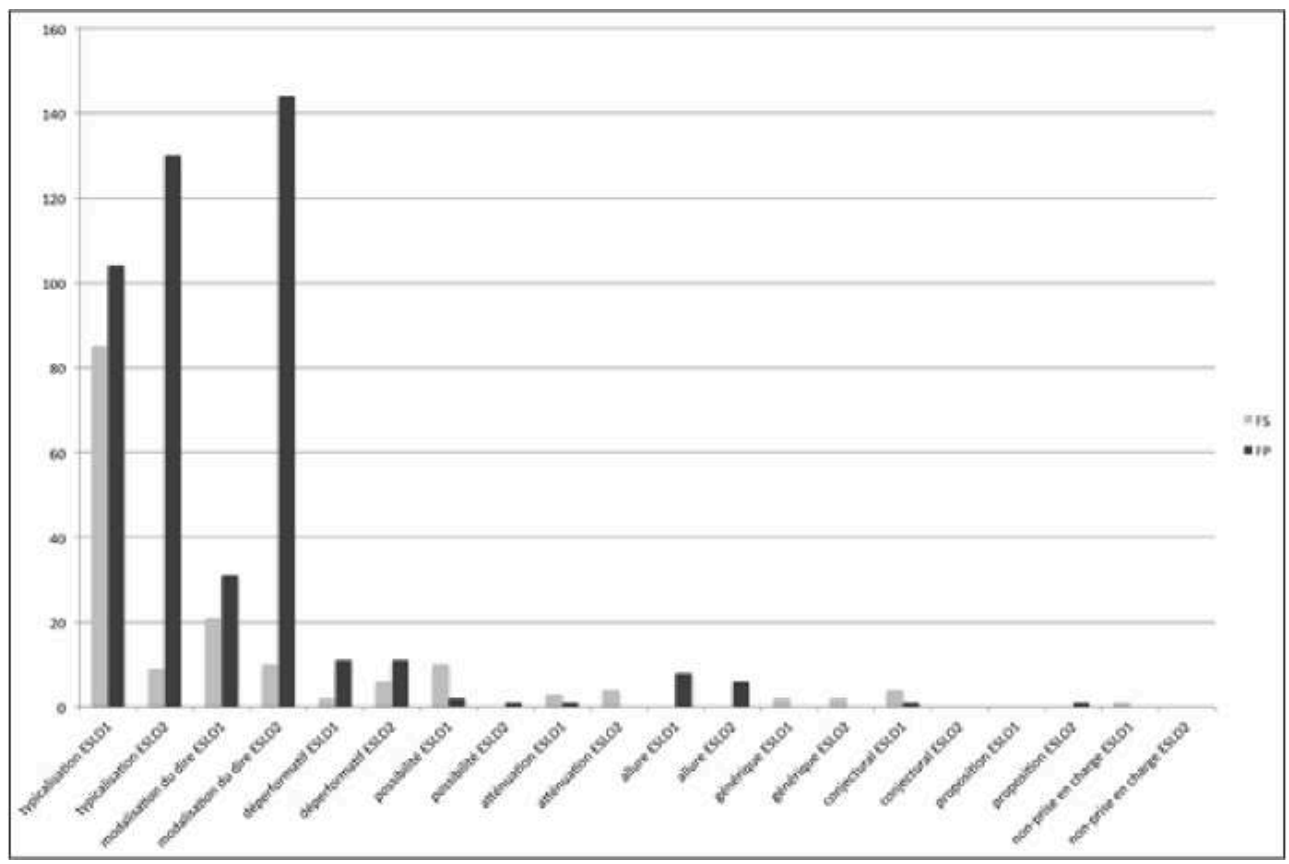

Figure 6 : Répartition des sous-spécifications des emplois modaux par tiroir verbal (FS vs FP) dans ESLO1 et ESLO2

\section{SOUS-SPÉCIFICATIONS SÉMANTIQUES DES EMPLOIS MODAUX : TENDANCES ET RAPPORTS DE FORCE}

Examinées de près, les tendances quantitatives observées dans le dernier graphique, montrent que, vis-à-vis de la question posée dans cette étude, à savoir la nature du lien diachronique entre le FP et le FS, aucune réponse simple ne peut être avancée. En effet, sans minimiser l'importance de la double tendance globale observée dès le début de la deuxième partie, à savoir un affaissement du FS et un essor du FP, lorsque nous observons le rapport FP/FS par le biais de la sous-spécification sémantique modale, nous arrivons à identifier, parallèlement à des cas de concurrence diachronique ayant abouti à une spécialisation du FP dans des domaines sémantiques disputés par le FS il y a encore 40 ans, des cas de complémentarité entre les deux tiroirs, garantissant au FS un maintien et même un quasi-monopole dans des niches sémantiques, voire des cas d'une évolution indépendante, où l'essor du FP ne semble pas se faire au détriment du FS.

\section{SPÉCIALISATION DU FP : TYPICALISATION}

Cette famille d'emplois de futur que nous proposons d'appeler typicalisation regroupe à la fois des cas habituellement décrits ailleurs comme des illustratifs (cf. Bres \& Labeau 2013), et des cas qui reçoivent parfois l'étiquette de générique (Damourette \& Pichon 
1911-1936). L'extrait suivant, qui comporte selon nous cette double dimension, en est un exemple :

ESLO2_ENT_1015_C_29

$\mathrm{Ch}$ : quand ils parlent euh ils ont des expressions particulières des expressions ? EW15 : oh bien oui hein euh moi-même je suis amenée quelquefois à les reprendre hein parce que je vous mets mes par exemple mes petites parisiennes hein elles ont elles ont quelquefois un vocabulaire euh hein euh c'est vrai on on va facilement prononcer des hein des des gros mots ou comme ça c'est hm hm hein tiroirs se spécialise, sans subir de concurrence, dans l'expression d'une valeur modale particulière non exprimable par l'autre. Nous trouvons les deux cas possibles : des domaines réservés au FP et d'autres au FS.

\section{FP : Allure extraordinaire}

41

Nous avons rangé dans cette catégorie les cas où la périphrase constitue, suivant la définition de Damourette \& Pichon (1911-1936 : \$2064), “un tour qui présente le phénomène comme ayant un caractère dérangeant par rapport à l'ordre attendu des 
choses"18, et qui par ailleurs n'a aucune valeur sémantique temporelle (même si dans certains emplois les deux valeurs peuvent se combiner, 1911-1936 : \$1655). Il est intéressant d'observer que, contrairement à ce que pouvaient laisser supposer certains travaux qui s'appuient essentiellement sur des occurrences de l'allure extraordinaire à l'affirmative (Bres \& Labeau 2013), la majorité des cas d'allure extraordinaire de notre corpus sont des énoncés négatifs, comme dans l'exemple suivant :

ESLO2_ENT_1023_C_36

Loc. A oh oh entre eux mais moi mes petits-enfants euh je veux pas qu'ils m'envoient des SMS et tout j'y comprends rien hein Loc. B ah bon

Loc. A ils parlent que comme ça hm vous allez pas me dire qu'après euh comme je dis à Carole et tout ça bah elle est à à cheval là-dessus

Nous ne recensons qu'un seul énoncé affirmatif, qui plus est dans ESLO1, ce qui laisse à penser qu'il puisse s'agir d'une forme désuète, ou peu fréquente dans les genres discursifs oraux examinés ${ }^{19}$ :

ESLO1_ENT_022_C_3

WL 512: je sais pas je suppose que le docteur me laissera quand même partir en vacances mais alors y a un un régime c' est ça aussi MD: ah

WL 512: allez donc dans les restaurants faire un régime comment vous voulez faire ?

Le décompte des occurrences donne la répartition suivante ${ }^{20}$ :

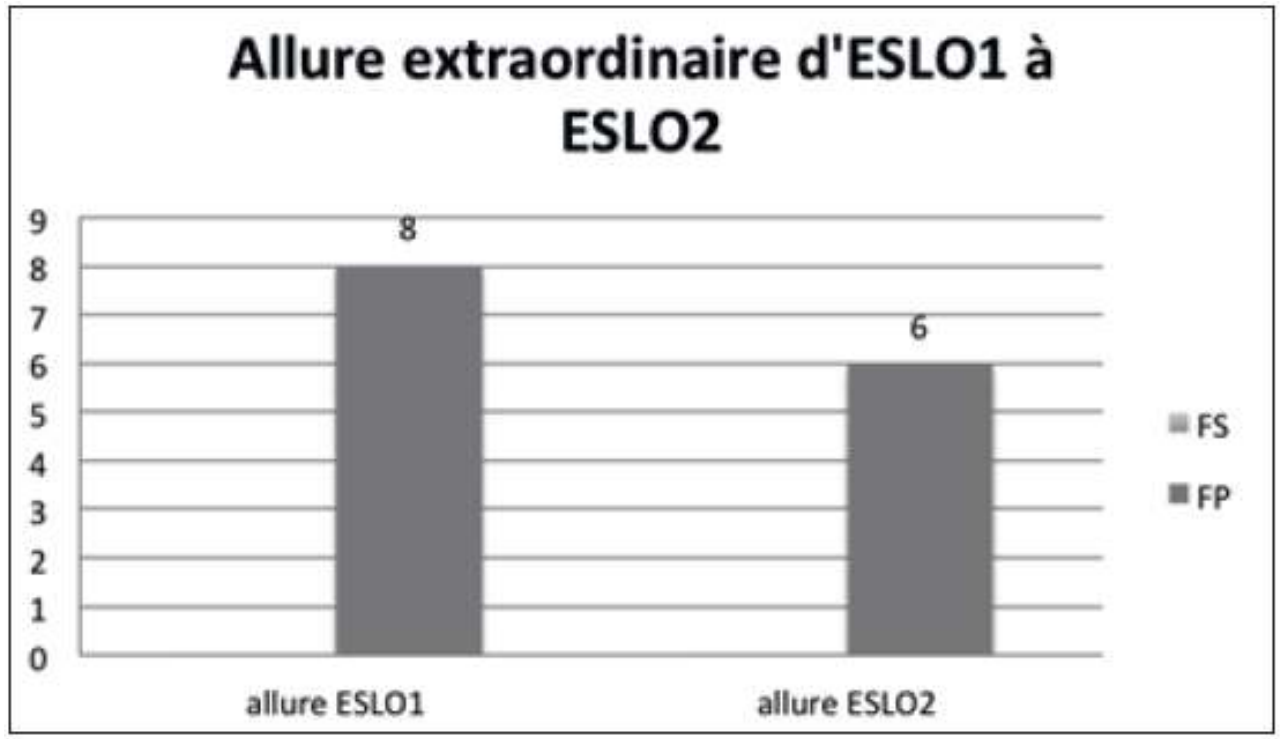

44 Figure 8 : Répartition FS/FP pour le type d'emploi allure extraordinaire d'ESLO 1 à ESLO2

45 Ainsi, malgré la faible fréquence de ce type d'emploi sur l'ensemble du corpus et une baisse relative d'ESLOl à ESLO2, l'allure extraordinaire est un domaine résolument propre au FP, sans aucune concurrence du FS en microdiachronie ${ }^{21}$.

\section{FS : Générique}

Parallèlement au cas qui vient d'être présenté, les emplois génériques illustrent un autre type de complémentarité entre les deux tiroirs, où le FS, ne rencontrant aucune concurrence du FP, se maintient dans des niches sémantiques. 
Ne confondant pas cette classe avec celle de typicalisation, nous réservons l'étiquette de générique à une toute petite série d'emplois où le futur présente le procès comme une prédication constante ${ }^{22}$, caractérisant une classe d'individus, comme dans l'extrait suivant :

ESLO2_CONF_4CPMEb_C_9

Augusto de Armas poète cubain auteur du remarquable livre en français Rimes byzantines évoque plusieurs fois la terrible phrase de son maître Théodore Banville nul étranger ne fera un vers français qui ait le sens commun

$\mathrm{Si}$, comparativement à d'autres types d'emplois, les occurrences concernées par ces emplois paraissent quantitativement négligeables dans le corpus, le maintien dans ESLO2 de ces emplois dans des proportions quasi identiques à ESLO1 nous parait mériter une attention particulière, et pourrait sans doute figurer parmi les éléments qui expliquent le maintien du FS mais dans des proportions peu importantes 2324 .

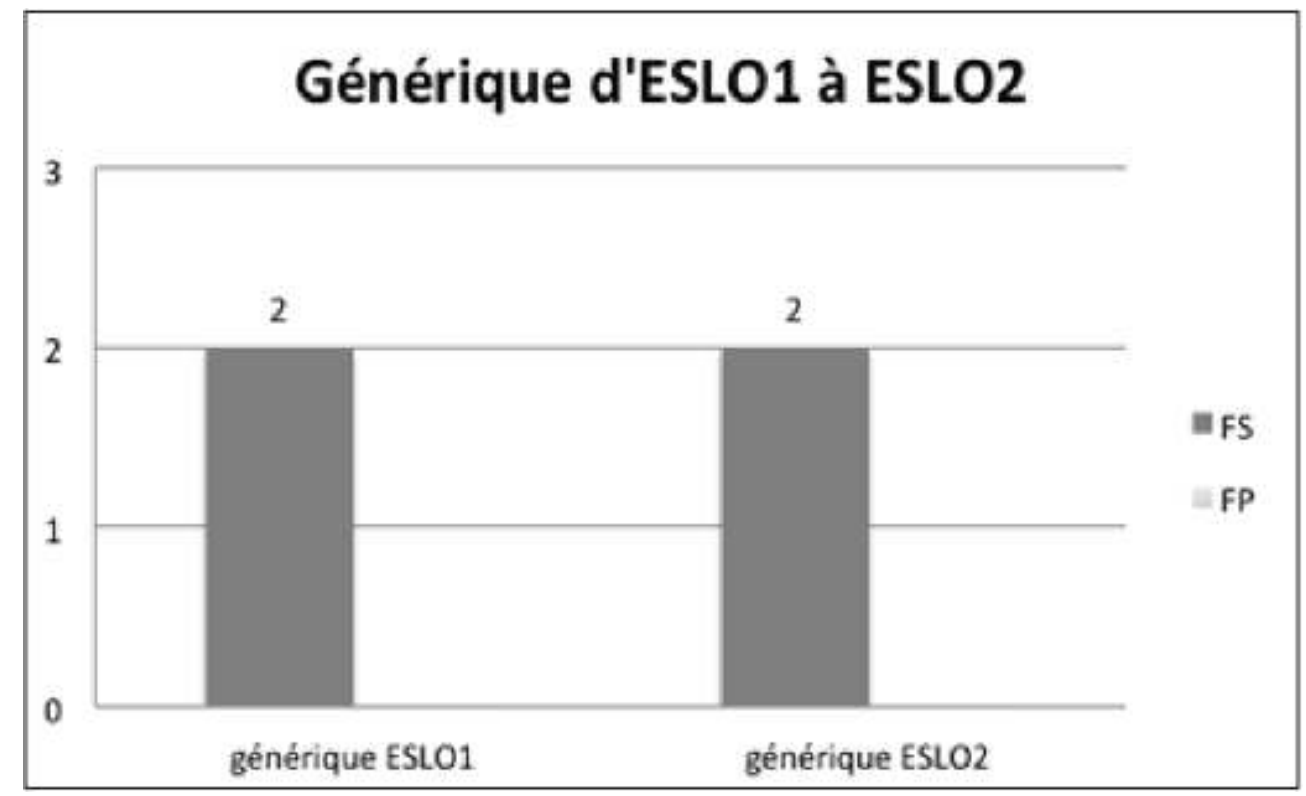

Figure 9 : Répartition FS/FP pour le type d'emploi générique d'ESLO 1 à ESLO2 ${ }^{24}$

\section{ÉVOLUTION INDÉPENDANTE : MODALISATION DU DiRE}

Un cas particulièrement intéressant de la relation FP/FS nous est fourni par la catégorie que nous appelons modalisation du dire par modulation d'un verbe de parole. Malgré sa proximité avec la catégorie atténuative et, partiellement, avec la catégorie des déperformatifs, elle s'en distingue par deux propriétés linguistiques particulières. D'une part, elle ne se rencontre qu'avec un champ lexical verbal bien spécifique et très restreint, i.e. les verbes de parole. Ceux-ci, ainsi qu'on le verra ci-dessous, correspondent quasi exclusivement à dire, non pas dans le sens premier de proférer mais dans celui d'énoncer et d'asserter, même si on y rencontre aussi quelques autres verbes à la fréquence marginale : demander (5 occurrences), citer (une occurrence) et parler (une occurrence). D'autre part, la modalité produite correspond à l'expression d'une réserve/distance vis-à-vis de ce qui est dit, présenté comme une approximation (relative à la quantification, à la catégorisation, au choix lexical, etc.) :

Ex. ESLO2_ENT_1001

BV 1: on a fait plongée mais c'était on était déçu 
ch_OB hm ah euh pour un baptême de plongée?

BV 1 ouais bah en même temps on a fait le tarif euh

ch_OB avec bouteille?

BV 1 de base donc euh c'était c'était on va dire euh dix minutes sous la petite flaque

d'essence euh du port où y a les trois quatre bateaux quoi

ch_OB ah ouais

BV 1 ouais deux trois poulpes des poissons mais [bruit de bouche] c'est pas terrible

Ex. ESLO2_ENT_1010

GK le fait de d'être en centre ville le côté où y a du monde euh ch_CD hm hm hm

GK du coup on on vit euh on $v$ - on vit une vie active quoi on va dire une vraie vie

ch_CD ouais voilà oui oui

GK de centre ville quoi euh c'est vrai que habiter à dix kilomètres d'Orléans et

rentrer chez soi tous les soirs et pas bouger ça euh

ESLO2_ENT_1085_C

RN488 : après je me suis retrouvée euh à la rue dirons-nous

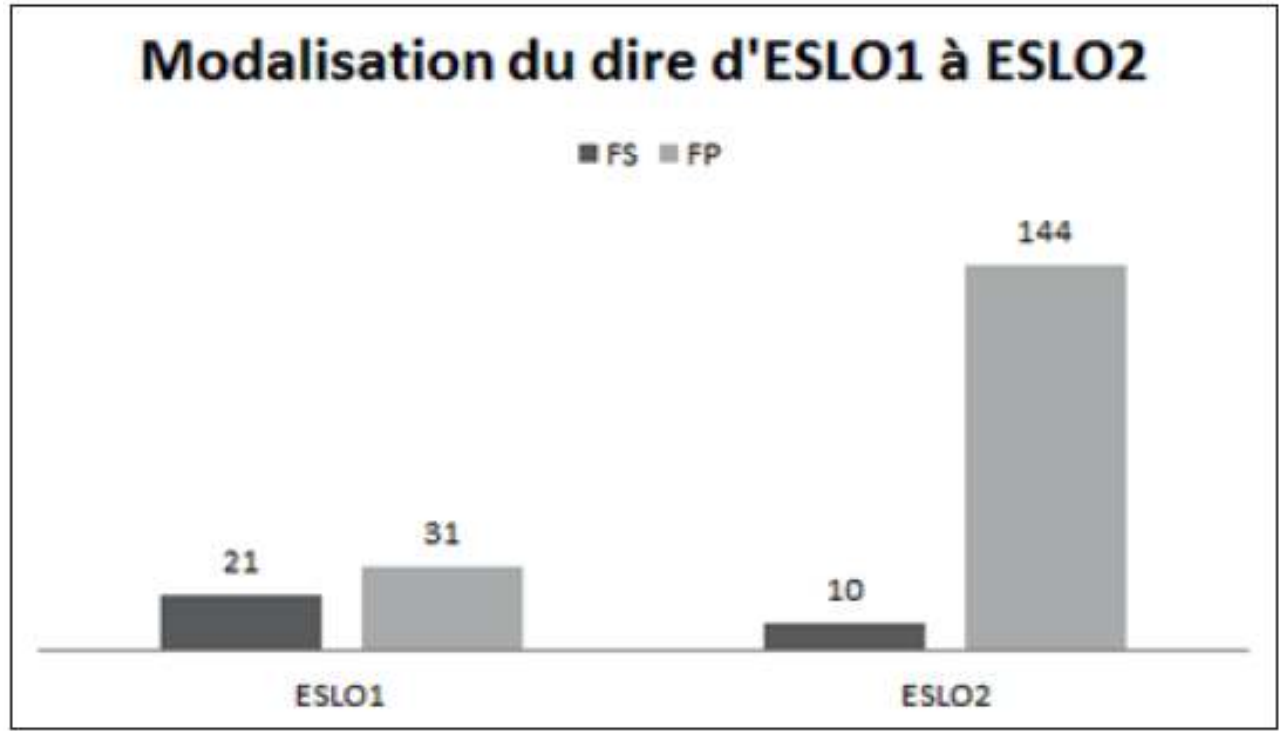

51 Figure 10 : Répartition FS/FP pour le type d'emploi modalisation du dire d'ESLO 1 à ESLO2

Comme on peut l'observer sur le graphique ci-dessus, la modalisation du dire constitue l'un des emplois où le FP a connu le plus d'essor. Si ce chiffre est intéressant en soi (il figure en bonne place parmi les emplois qui expliquent l'essor du FP en général), il interpelle lorsqu'on le compare à l'évolution du FS pour le même type d'emploi : là où la fréquence du FS ne baisse que de 53\%, celle du FP a été multipliée par plus de 4,5. En bref, le FP gagne bien plus que ce que le FS perd. Cette "anomalie statistique" s'explique par l'éruption, repérée depuis peu dans certains travaux (cf. Steuckardt 2014 par exemple), de la forme on va dire, comme on peut le voir dans le tableau suivant :

\begin{tabular}{|l|l|l|}
\hline & ESLO1 & ESLO2 \\
\hline aller + INF & 715 & 1204 \\
\hline aller + dire & 58 & 179 \\
\hline on va dire (de type modalisation) & 4 & 123 \\
\hline
\end{tabular}



FP et FS ayant reçu cette étiquette peuvent être glosés pour la plupart par une paraphrase à l'impératif, la forme étant utilisée pour éviter une formulation à caractère injonctif, que ce soit à des fins pragmatiques d'atténuation, ou simplement pour asserter la quasi certitude de la réalisation du procès qui fait l'objet de la volonté de l'énonciateur (cas illustrés respectivement par les exemples qui suivent au FP) :

ESLO2_ENT_1057_C

UZ57 : et après et ben j'ai été figurez-vous que j'ai été travailler dans un cinéma et que j'y suis toujours ch_LA11: d'accord UZ57: voilà

ch_LA11: hm hm c'est lequel?

UZ57: euh Place d'Arc à UGC

ch_LA11: d'accord et alors euh on va tr- on va on va travailler on va UZ57: hm ch_LA11: nous sommes en train de travailler mais euh vous allez nous décrire un petit peu en quoi consiste euh votre euh travail UZ57: ma fonction de maintenant actuelle ou ? ouais ? ch_LA11: euh oui oui

UZ57: hm alors je suis comment on appelle ça chef d'équipe

ESLO1_REPAS_273_B_21

Hervé tu vas être bien élevé et fermer la porte hein

La distribution des formes du FS/FP d'ESLO1 à ESLO2 est la suivante :

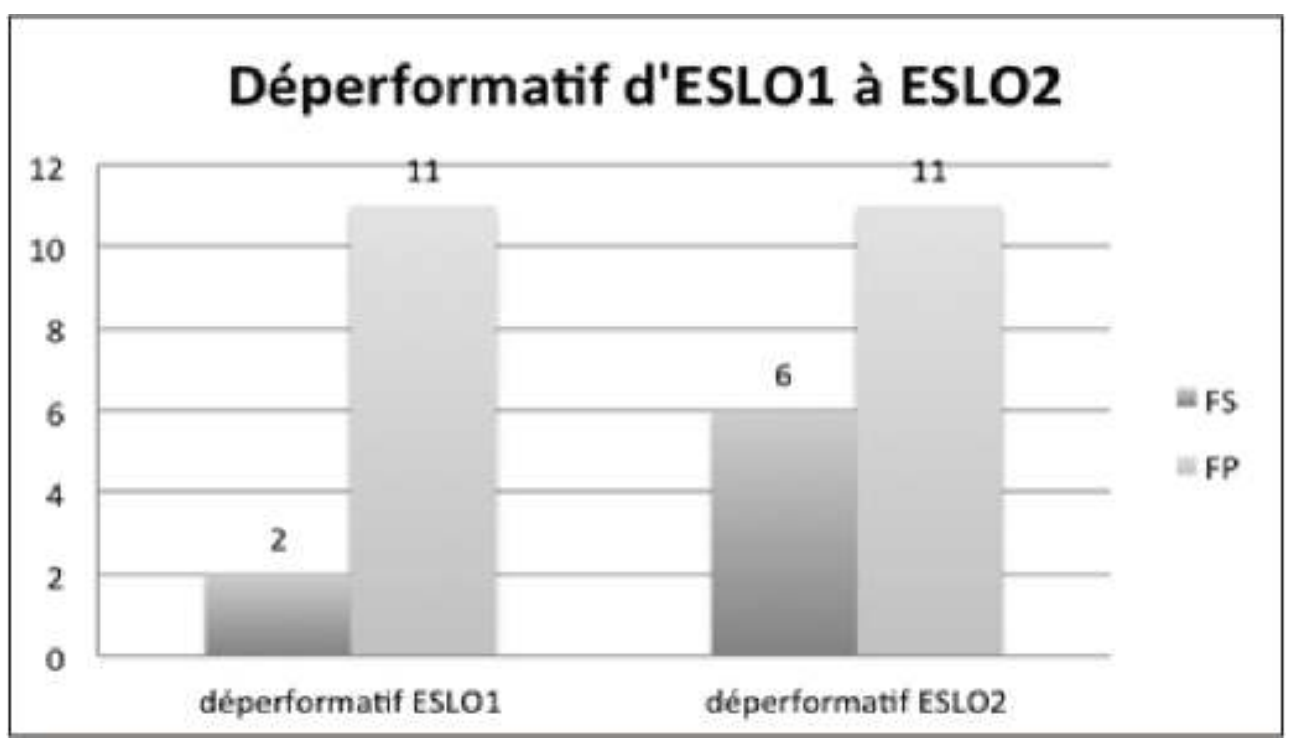



un rapport intéressant. La tendance générale est celle d'un maintien du FP s'accompagnant d'une progression du FS, ce dernier étant 3 fois plus représenté dans cet emploi dans ESLO2 que dans ESLO1. Il semble donc qu'il s'agit d'un cas de concurrence entre les deux tiroirs verbaux, hypothèse qui repose sur l'idée que les deux tiroirs verbaux se disputent le même terrain, comme c'est le cas dans ESLO2. Pourtant, si l'on examine de près les données du sous-corpus ESLO2, on peut faire un constat intéressant : tandis que 6 des 11 cas de FP déperformatifs apparaissent dans un des genres interactionnels de contrôle, i.e. les repas en famille ou entre amis, 5 des 6 cas de FS déperformatifs sont répertoriés dans l'autre genre de contrôle, les conférences $^{27}$. L'hypothèse d'une réelle concurrence entre les deux formes pour ce type d'emplois est donc à relativiser, au profit de celle d'une complémentarité diaphasique, le FP déperformatif s'ancrant dans la proximité, le FS dans la distance communicative. Ici, nos observations vont dans le sens des travaux de Arrivé, Gadet \& Galmiche (1986), ou Maingueneau (1994), qui soutiennent l'hypothèse diaphasique pour expliquer la distribution FS/FP ainsi que l'essor du FP en vernaculaire. On constate donc que l'explication diaphasique peut bel et bien être mobilisée pour certains emplois, mais l'on se gardera de toute généralisation sur l'ensemble de la distribution FS/FP.

\section{CONCLUSION ET PERSPECTIVES}

61 Au terme de cette étude sur corpus, il nous parait impossible d'adopter exclusivement une seule des hypothèses qui s'affrontent dans les travaux qui tentent de décrire le rapport entre les deux formes du futur, chacune d'entre elles ne parvenant à expliquer qu'une facette d'un rapport qui s'avère finalement assez complexe. Cela montre que seul un corpus d'ampleur suffisante et composé de données situées peut permettre une description quantitative et qualitative nuancée et différenciée, évitant l'écueil d'une explication simplificatrice. Certains des travaux dans le champ traitent de ces phénomènes parfois isolément, sur des données non situées, courant le risque de perdre de vue le caractère multidimensionnel de la variation.

62 Les résultats obtenus indiquent par ailleurs des tendances qui ne permettent pas de valider des hypothèses comme celle de Fleischman (1982), ou encore Bybee, Pagliuca et Perkins (1991) selon lesquelles le renouvellement des formes du futur dans plusieurs langues romanes, cyclique ${ }^{28}$, serait dû à une évolution de la forme future vers une modalisation, ce qui aurait pour conséquence d'appeler l'apparition d'un nouveau temps verbal pour l'expression de la futurité. Le processus de modalisation jouerait donc un rôle moteur. Or, si nos données montrent une progression du FP globalement homogène (augmentation en proportions similaires dans les domaines de la modalité et de la futurité), le recul du FS en revanche est plus important dans le domaine de la modalité que de la futurité. Il n'est donc pas possible, au regard de nos résultats, d'imputer la progression du FP à une expansion modale du FS.

Enfin, il nous semble que l'hypothèse aspectuelle, défendue par ailleurs par certains linguistes ${ }^{29}$ et que nous n'avons pas pu interroger ici, mérite quelque attention. Qu'il s'agisse de la question de l'opposition entre statif/non statif (Bilger 2001, Jeanjean 1988, Maingueneau 1994, Franckel 1989), du lien avec le présent (present/current relevance 
Fleischman 1983) ou de la prospection, différentes facettes aspecto-temporelles sont susceptibles d'entrer en jeu dans le rapport de force entre les FS et FP ayant reçu dans notre typologie l'étiquette $\mathrm{f}$. Un test de substituabilité FP - FS pourrait notamment être mené pour une observation détaillée et une sous-spécification de ces emplois temporels. L'hypothèse d'une concurrence des formes dans le domaine de la stricte temporalité corrélée à une complémentarité dans le domaine de l'aspectualité pourrait ainsi constituer un prochain terrain d'investigation sur les ESLO.

\section{BIBLIOGRAPHIE}

Abouda, L. (2004). « Deux types d'imparfait atténuatif », Langue française, 142, 58-74.

Abouda, L. (2015). Syntaxe et Sémantique en corpus. Du temps et de la modalité en français oral, Habilitation à Diriger des Recherches, Université d'Orléans.

Abouda, L. \& Baude, O. (2007). «Constituer et exploiter un grand corpus oral : choix et enjeux théoriques. Le cas des Eslo ", in F. Rastier et M. Ballabriga (dir.), Corpus en Lettres et Sciences sociales. Des documents numériques à l'interprétation, Actes du XXVIIe Colloque d'Albi « Langages et Signification »,161-168.

Arrivé, M., Gadet, F. \& Galmiche, M. (1986). La Grammaire d'aujourd'hui. Guide alphabétique de linguistique française, Flammarion.

Barcelo, G. J. (2007). « Le(s) futur(s) dans les langues romanes : évolution linéaire ou cyclique? », Sémantique et diachronie du système verbal français, Cahiers Chronos, 16, Amsterdam, New York, NY, 47-62.

Biber, D. (2009).“Corpus-Based and Corpus-driven Analyses of Language Variation and Use” in: Bernd Heine and Heiko Narrog (ed.), The Oxford Handbook of Linguistic Analysis, Oxford University Press.

Bilger, M. (2001). « Retour sur le futur dans les corpus de français parlé », RSFP, 16, Université de Provence, 177-189.

Bres, J. \& Labeau, E. (2013). « Allez donc sortir des sentiers battus! La production de l'effet de sens extraordinaire par aller et venir », Journal of French Language Studies, 23,151-177.

Bybee, J.L., Pagliuca, W. \& Perkins, R.D. (1991). "Back to the future”, in. Traugott, E.C. \& Heine, B. (eds). Approaches to the grammaticalization, II, Amsterdam/ Philadephia, John Benjamins Publishing Company, 17-58.

Celle, A. (2005). "The French future tense and English will as markers of epistemic modality", Languages in Contrast, 5,181-218.

Collier, M. (2013). “The aller Perfect”, in Labeau, E., Bres, J. (ed.), Evolution in romance verbal systems, Peter Lang, 203-220.

Confais, J.-P. (1990). Temps, Mode, Aspect, Toulouse,Presses Universitaires de Mirail.

Damourette, J. et Pichon, E. (1911-1936). Des mots à la pensée. Essai de Grammaire de la Langue Française, tome V, Editions d'Artrey. 
Desahaies, D. \& Laforge, E. (1981). « Le futur simple et le futur proche dans le français parlé dans la ville de Québec », Langues et Linguistique, 7, 21-37.

Engel, D. M. (1996). «L'expression du temps et la variation linguistique », Revue Romane, 31,215-233.

ESLO_V1 : Enquêtes Sociolinguistiques à Orléans, 2013, LLL-UMR 7270, Université d'Orléans, http://eslo.huma-num.fr

Emirkanian, L. ; Sankoff, D. (1986). « Le futur simple et le futur périphrastique dans le français parlé » in Morphosyntaxe des langues romanes, Actes du XVII ${ }^{\mathrm{e}}$ Colloque international de linguistique et de philologie romanes, Aix-en-Provence,397-407.

Fleischman, S. (1982). The future in thought and language, New York, Cambridge University Press.

Fleischman, S. (1983). "From pragmatics to grammar: diachronie reflections on complex pasts and futures in Romance”, Lingua, 60, 183-214.

Franckel, J-J. (1984). « Futur simple et futur proche », Le Français dans le monde, 182, 65-70.

Fleury, S. \& Branca, S. (2010). «Une expérience de collaboration entre linguiste et spécialiste de TAL : L'exploitation du corpus CFPP 2000 en vue d'un travail sur l'alternance Futur simple / Futur périphrastique », Cahiers AFLS, Volume 16(1).

Gosselin, L. (1996). Sémantique de la référence temporelle en français, Louvain-laNeuve, Duculot. Gosselin, L. (2005), Temporalité et modalité, (postface de B. Victorri), Bruxelles, Duculot-de Boeck, col. Recherches.

Gosselin, L. (2010). Les modalités en français. La validation des représentations, Rodopi, coll. Etudes Chronos, Amsterdam / New York, 373-390.

Gougenheim, G. (1929). Étude sur les périphrases verbales de la langue française, A.-G. Nizet, Paris, 1971.

Grevisse, M. \& Goosse, A. (2008). Le Bon usage, $14^{\mathrm{e}}$ édition, De Boeck \& Duculot, Bruxelles.

Jeanjean, C. (1988). «Le futur simple et le futur périphrastique en français parlé : Étude distributionnelle », in C. Blanche-Benveniste et al., Grammaire et histoire de la grammaire : Hommage à la mémoire de Jean Stéfanini. PU de Provence, 235-257.

Lansari, L. (2009). Linguistique contrastive et traduction. Les périphrases verbales aller + infinitif et be going to, Ophrys.

Laurendeau, P. (2000). «L'alternance futur simple/futur périphrastique : une hypothèse modale ", Verbum, tome 22, n³, PU de Nancy, 277-292.

Maingueneau, D. (1994). L'énonciation en linguistique française, $2^{e}$ édition (1999), Hachette.

Martin, R. (1981). « Le Futur : temps linéaire ou temps ramifié ? », Langages, 64, 81-92.

Mauger, G. (1968). Grammaire pratique du français d'aujourd'hui, Paris, Hachette.

Mullineaux, L.A. \& Blanc, M. H. A. (1982). “The problem of classifying the population sample in the socio linguistic survey of Orléans (1969) in terms of socio-economic, social and educational categories", Review of Applied Linguistics, 55, 3-37.

Nadasti, T., Mougeon, R., Rehner, K. (2003). « Emploi du futur dans le français parlé des élèves d'immersion française », Journal of French Language Studies, 13, 195-219. 
Riegel, M., Pellat, J.-Ch. \& Rioul, R. (1994). Grammaire méthodique du français, Presses Universitaires de France.

Rocchetti, A. (2004), « La syntaxe du futur et du conditionnel dans les langues romanes : le futur après «si» et le futur dans le passé », in Luquet, G., Rocchetti, A. (ed.), Regards sur la syntaxe historique des langues romanes, Paris : Presses de l'Université Paris-III, 67-106.

Steuckardt, A. (2014). « Polyphonie et médiativité dans un marqueur émergent : on va dire », in Anscombre J.-C., Oppermann-Marsaux E., Rodriguez Somolinos A. (éds.) : Médiativité, polyphonie et modalité en français: études synchroniques et diachronique, Paris : Presses de la Sorbonne Nouvelle, 67-84.

Togeby, K. (1982). Grammaire française, Copenhague, Akademisk Forlag, vol.2-3.

Wilmet, M. (2007). Grammaire critique du français, $4^{\mathrm{e}}$ édition, de Boeck \& Larcier, Bruxelles.

\section{NOTES}

1. http://eslo.huma-num.fr/

2. Nous avons repris les catégories de l'échelle Alix Mullineaux (échelle AM, cf. Mullineaux \& Blanc 1982), utilisés pour les entretiens ESLO1, en les adaptant au cas par cas aux profils ESLO2. Pour une présentation détaillée de ce sous-corpus et une discussion des critères ayant présidé à sa constitution, voir Abouda (2015 : 111 sq. et $137-141)$.

3. Nous sommes bien conscients que la comparabilité reste relative du point de vue qualitatif (évolution des techniques d'entretien, statuts différents des enquêteurs, etc.).

4. http://textometrie.ens-lyon.fr/

5. http://www.cis.uni-muenchen.de/ schmid/tools/TreeTagger/

6. La syntaxe de la requête CQL pour le FP : [frlemma="aller"\&frpos="VER:pres"][] \{0,5\}[frpos="VER:infi"] et pour le FS : [frpos="VER:futu"].

7. Cette distribution s'éclaire quelquefois mieux lorsqu'on croise ces informations avec des métadonnées relatives au genre interactionnel et à l'activité de communication en cours, voir $\$ 3.4$ ci-dessous.

8. Encore que l'extension exacte de la classe des emplois temporels et celle des emplois modaux varie selon les auteurs. Pour nous, l'emploi temporel est un emploi qui situe le procès dans un moment ultérieur à un repère référentiel, qui est généralement le moment de l'énonciation, mais qui peut aussi correspondre à un repère distinct, comme c'est le cas pour le futur historique :

ESLO2_CONF_4CPMEb_C_11:

le récit se clôt là où il avait commencé à la frontière franc- franco-espagnole en Hendaye l'endroit où le père de Jacques avait disparu et où Jacques mourra aussi incapable de dépasser les clivages linguistiques et culturels que sa mère avait vainement essayé de supprimer en lui ou encore pour le futur relatif :

ESLO2_CONF_5FLb_C_3 :

Platon mais aussi d'autres évoquent le symposium grec où les convives se passent un cratère où dans lequel il y a de l'eau et du vin et ils font l'éloge de l'amour mais pas seulement n'est-ce pas lors de ces banquets et en quelque sorte ils se lancent des défis et ils essayent de finalement de de faire le l'éloge qui sera le le plus apprécié par tous hein

9. Notre vision, concernant ces emplois mixtes, rejoint ainsi partiellement celle que défend Maingueneau (1994) à propos du futur dans son ensemble, vu par l'auteur comme porteur d'une tension modale systématique. Pour nous, s'il existe des emplois mixtes où la futurité s'accompagne d'un certain nombre de traits modaux, il existe parallèlement des emplois exclusivement temporels et des emplois exclusivement modaux. 
10. Cf. Damourette \& Pichon (1911-1936 : \$1655) qui identifient des cas mixtes alliant ultériorité et modalité extraordinaire.

11. Voir à ce propos Confais (1990), et surtout Gosselin (2005) qui critique cette dichotomie exclusive temps-modalité.

12. Maingueneau voit dans les combinaisons de tu + futur de ce type l'expression d'une prédiction avec une modalité de nécessité (implicite).

13. Les catégories modale et temporelle n'étant pas vues ici comme mutuellement exclusives, on ne retiendra pas la commutation avec un présent comme test pertinent pour l'identification du trait modal affectant un tiroir futur. On aura d'ailleurs remarqué que la commutation avec un présent ne fonctionne pas dans le cas présent.

14. Ces résultats entrent en contradiction avec ceux obtenus dans des études récentes sur des corpus de français parlé (cf. Bilger 2001, Jeanjean 1988), mais confirment les tendances dégagées par Emirkanian \& Sankoff (1986).

15. Ces données ne semblent pas corroborer les hypothèses de Fleischman (1982) qui explique le renouvellement des formes du futur en diachronie par la progression des emplois modaux qui rendraient nécessaire la mobilisation d'une nouvelle forme pour l'expression de la futurité.

16. Cela rejoint les observations de Lansari (2009:53) qui prend note du caractère à la fois spécifique et générique de la périphrase aller + inf. D'après elle, le FP permet de réaliser une induction à partir d'observables pour établir un principe général. Elle cite l'exemple suivant, que nous rangerions précisément dans la catégorie typicalisation : certes un programme comptant de 1 à 10 va tourner correctement dans une montre ou un superordinateur. La même analyse peut s'appliquer à l'extrait suivant tiré de notre corpus : ESLO1_ENT_121_C_32

A: et quand vous écrivez à des amis est-ce que vous faites un brouillon?

B: non non non euh si j'écris euh pour mon travail oui je vais faire un brouillon et je vais rechercher mon vocabulaire si j'écris à des amis j'aurais toujours tendance à prendre un petit peu un style euh comme on croise souvent qu'on appelle télégraphique En formulant le procès au FP, le locuteur B généralise un comportement à la fois spécifique et habituel sans pour autant se situer dans une visée pragmatique d'illustration.

17. Notre analyse rejoint donc en partie celle de Rebotier (ce volume) qui considère l'illustratif comme un cas particulier de générique.

18. D'autres auteurs, plus récemment, décrivent de manière similaire le sémantisme du tour. Lansari (2009 : 85 sqq.) considère qu'il permet « l'attribution et rejet d'une intention, grâce à une opération fondamentale d'évaluation négative exploitée dans le cadre d'une relation intersubjective ».

19. Notons que la plupart des exemples à l'affirmative cités par Bres \& Labeau (2013) relèvent du domaine littéraire.

20. On ne recense aucune occurrence au FS ce qui explique l'absence de colonne FS à gauche des colonnes FP sur le graphique.

21. Nous ne trouvons d'ailleurs pas d'exemple dans notre corpus qui corresponde à l'emploi décrit par Damourette \& Pichon (1911-1936 : 824) d'extraordinaire dit "impur" au futur (qui s'oppose à l'extraordinaire pur avec aller + inf au présent) : je n'irai pas payer vingt-deux francs une culotte quand je peux avoir pour dix-neuf sous un superbe couteau à trois lames (Courteline. Les bonnes occasions ; CEuvres, t. V, p.278).

22. Si nous additionnons les étiquettes $m$ et $\mathrm{fm}$, le nombre d'occurrences spécifiées génériques atteint 12 (toutes au FS). Il est significatif de constater que sur 12 énoncés, 4 se combinent avec l'adverbe toujours, et un avec l'adverbe jamais. D'autres quantificateurs généralisant peuvent affecter le sujet, comme nul (1), et rien (1).

23. Une analyse analogue peut être défendue à propos des emplois atténuatifs (que nous distinguons à la fois des déperformatifs et des cas de modalisation du dire par un verbe de parole, cf. infra) en tout comparables aux emplois génériques (absence d'évolution quantitative, emploi peu fréquent dans le corpus, 
et une sous-spécification quasi exclusivement au FS) : nous notons 4 emplois de ce type dans ESLO1, et 4 dans ESLO2. Sur les 8 occurrences, 7 sont au FS, et 1 au FP (appartenant à ESLO1).

24. On ne recense aucune occurrence au FP ce qui explique l'absence de colonne FP à droite des colonnes FS sur le graphique.

25. On peut constater que la substitution de on va dire par disons ne pose pas le moindre problème dans les trois exemples fournis ci-dessus pour illustrer ce type modal au FP.

26. La déperformativité (Abouda 2004) est un procédé, marqué sur le plan morphosyntaxique, de neutralisation formelle d'un acte de langage exprimé par l'énoncé performatif correspondant. Dans nos exemples, certains emplois du futur se présentent comme un substitut naturel à des emplois injonctifs à l'impératif.

27. Les genres de contrôle constituent respectivement $10 \%$ du corpus sélectionné.

28. Le futur synthétique du latin cantabo se voit en effet remplacé par la forme analytique cantare habeo qui se synthétise en chanterai avant d'être elle-même concurrencée depuis une époque plus récente par la périphrase aller + inf.

29. Pour Fleischman (1983) par exemple, le mécanisme à l'œuvre dans le changement relève également du domaine aspectuel, plus exactement du passage de l'aspectualité à la temporalité, le FP, à l'origine périphrase prospective, en venant à exprimer la futurité. Cela est vrai également pour le passage de cantare habeo à chanterai.

\section{RÉSUMÉS}

La présente étude traite du rapport synchronique et micro-diachronique entre les formes synthétique et analytique du futur en français moderne, sur la base d'un corpus oral d'un million de mots, collecté à 40 ans d'intervalle (ESLO1 (1968-1971) et ESLO2 (2008-)). Les 3370 occurrences de futur identifiées ont été annotées, afin de déterminer le type d'emploi (modal, futural et mixte) ainsi que la sous-spécification sémantique modale (atténuation, typicalisation, modalisation du dire, etc.). Notre regard sera focalisé ici sur le rapport FP (futur périphrastique)/ FS (futur simple) en relation avec la variable modale. Si les tendances quantitatives montrent d'ESLO1 à ESLO2 une forte augmentation du FP et un affaissement du FS, l'examen plus précis du rapport de force entre les deux tiroirs à la lumière de la variable modale nous fait découvrir une situation contrastée où coexistent différents cas de figure, allant de la concurrence diachronique ayant abouti au triomphe du FP dans certains emplois, à la complémentarité, garantissant à chacun des deux tiroirs un domaine sémantique réservé (par exemple, l'emploi générique pour le FS, l'allure extraordinaire pour le FP), voire à une évolution indépendante montrant un FP qui progresse non pas au détriment du FS mais au dépens d'autres formes linguistiques.

This study deals with the synchronic and micro-diachronic relation be- tween synthetic (SF) and periphrastic (PF) future tense in modern French, in a one million words corpus of spoken French, that was collected with an interval of 40 years (ESLO1 (1968-1971) and ESLO2 (2008-)). We have annotated the 3370 instances of future tense (SF and PF) contained in the corpus, according to a first semantic différentiation between modal, futural or both modal-futural uses. A second semantic annotation level was defined for all tokens of modal future, according to a set of semantic sub-specification types (mitigation, typicalization, speech modalization, etc.). We will focus here on the distribution of periphrastic future vs. synthetic future in their modal use, in order to understand the first frequency count that have shown a strong decrease of FS in this 
use. The systematic review of the modal PF and SF shows a contrasted situation: regarding the special case of the sub-spectification typicalization, SF and PF undergo diachronic competition that leads to a much higher frequency of FP in ESLO2; in other configurations, each tense remains more frequent in a specific semantic area (SF for generic vs. PF for the so called allure extraordinairemirative); finally, in the case of speech modalization, the increased frequency of PF seems to be independent from the evolution of FS, with PF replacing other linguistic forms than SF.

INDEX

Index géographique : France

Mots-clés : futur simple, futur périphrastique, français, corpus oral, micro-diachronie, modalités

\section{AUTEURS}

\section{LOTFI ABOUDA}

LLL (UMR 7270) - Université d'Orléans

MARIE SKROVEC

LLL (UMR 7270) - Université d'Orléans 